\title{
Research on seismic properties of steel reinforced PP ECC column
}

\author{
Ming-xin $\mathrm{Yu}^{1 *}$, Lin Wang ${ }^{2}$, Jia-huan $\mathrm{Yu}^{3}$, Nan Yang ${ }^{1}$ \\ ${ }^{1}$ Department of Civil Engineering, Shenyang Urban Construction University, Shenyang, Liaoning, 110167, China \\ ${ }^{2}$ Liaoning Urban and Rural Construction Planning and Design Institute Co. Ltd, Shenyang, Liaoning, 110006, China \\ ${ }^{3}$ School of Civil Engineering, Shenyang Jianzhu University, Shenyang, Liaoning, 110168, China;
}

\begin{abstract}
The mechanical property of steel reinforced PP ECC columns under reverse cyclic load is investigated and results are presented in this paper. The influence of reinforcement ratio, curing age and volume fraction of PP fiber on load bearing capacity, energy dissipation and stiffness degradation is investigated. The results highlighted the positive contribution of PP ECC to enhance strength and energy dissipation capacity which is important to evaluate the performance of structures subjected to reverse cyclic loads. According to the experimental study on mechanical behavior of steel reinforced PP ECC columns under reverse cyclic loading, the formula of model parameters related to reinforcement ratio are proposed, it is founded that the restoring force model established is of a certain degree of adaptability.
\end{abstract}

\section{Introduction}

Because of the high cost of Engineering cement-based composites (PVA ECC), it is not conducive to marketization in the research of PVA ECC at present. Modified polypropylene (PP) fibers and optimized mix ratio were used to produce super-strength and toughness concrete (PP ECC) in this study. The cost of PP ECC is much lower than that of traditional ECC, and it has good engineering prospects. PP ECC overcomes the main shortcomings of traditional concrete, solves many problems encountered in the application of concrete in engineering circles, and it is an ideal substitute material for concrete. PP ECC has been fully localized, breaking through the price bottleneck, and the construction technology is simple at present ${ }^{[1]-[4]}$.

According to the excellent performance of PP ECC, it is possible to use PP ECC to replace ordinary concrete materials and reinforcement to work together. Because of PP ECC is different from concrete and has strong deformation capacity, it still bears loads after cracking. Therefore, it is necessary to conduct in-depth study on this material structure, especially its seismic performance.

\section{Testing situation}

\subsection{Specimen and loading.}

The parameters of the specimens are shown in Table 1, and the reinforcement in size is shown in Figure 1. Based on the measured stress-strain curves of compression and tension of PP ECC ${ }^{[5]}$, as shown in Fig. 2, the data are used in this study.

Table 1 Parameters of specimen

\begin{tabular}{ccccccc}
\hline No. & Axial pressure ratio & $\begin{array}{c}\text { PP content } \\
(\%)\end{array}$ & $\begin{array}{c}\text { Reinforcement } \\
\text { diameter(mm) }\end{array}$ & $\begin{array}{c}\text { Reinforcement ratio } \\
(\%)\end{array}$ & $\begin{array}{c}f_{c u} \\
(\mathrm{MPa})\end{array}$ & $\begin{array}{c}\text { Age } \\
(\mathrm{day})\end{array}$ \\
\hline $\mathrm{C} 1$ & 0.8 & 1.5 & 18 & 4.10 & 56 & 60 \\
$\mathrm{C} 2$ & 0.8 & 1.5 & 22 & 6.18 & 56 & 60 \\
$\mathrm{C} 3$ & 0.8 & 1.5 & 22 & 6.18 & 40 & 30 \\
$\mathrm{C} 4$ & 0.8 & 2.0 & 22 & 6.18 & 56 & 60 \\
$\mathrm{C} 5$ & 0.8 & 1.5 & 25 & 6.18 & 56 & 60 \\
$\mathrm{C} 6$ & 0.8 & 0 & 22 & & 60 & 60 \\
\hline
\end{tabular}




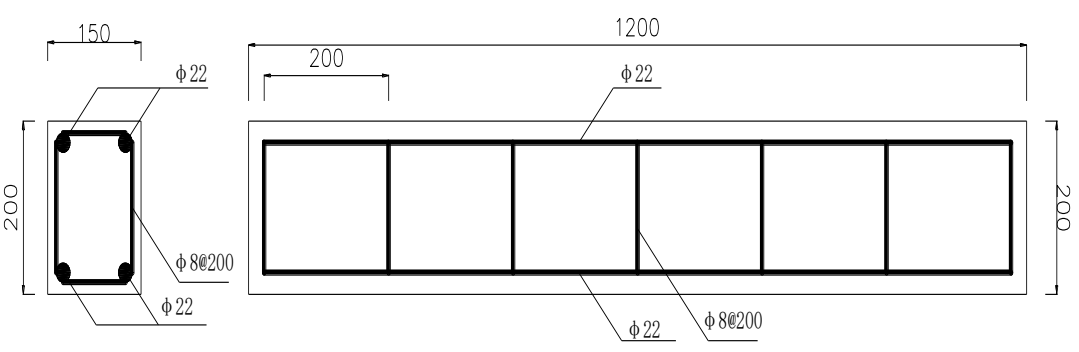

Figure 1 Dimensions and reinforcement details of specimens
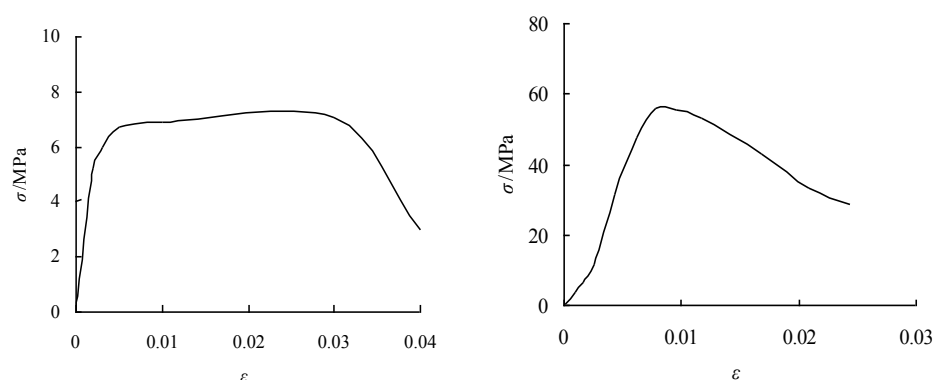

Figure 2 Stress-strain curves of PP ECC

The hysteretic test is used in the study ${ }^{[6]}$. The loading device is shown in Figure 3. The test axial pressure ratio

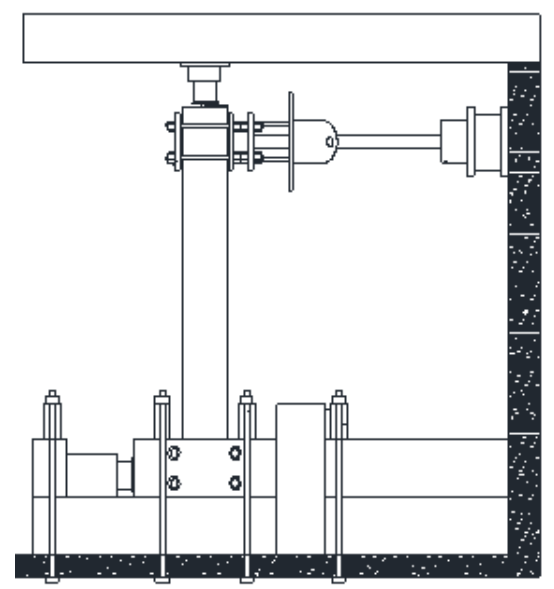

Figure 3 Setup of test

\subsection{Destruction phenomenon}

Cracks appear on the surface of all reinforced PP ECC columns at the beginning of loading, but the cracks are small, as shown in Fig.5 (a); with the increase of loading displacement, cracks develop slowly, as shown in Fig.5 (b); when the load reaches $60 \%$ ultimate bearing capacity, the number of cracks increases; when the load reaches $80 \%$ ultimate bearing capacity, the number of cracks remains unchanged, as shown in Fig.5 (c); When the load reaches the ultimate bearing capacity, the crack width near the fixed end increases and the main crack appears. The sound of the fiber being pulled out can be heard. is fixed, and the specific loading system is shown in Figure 4.

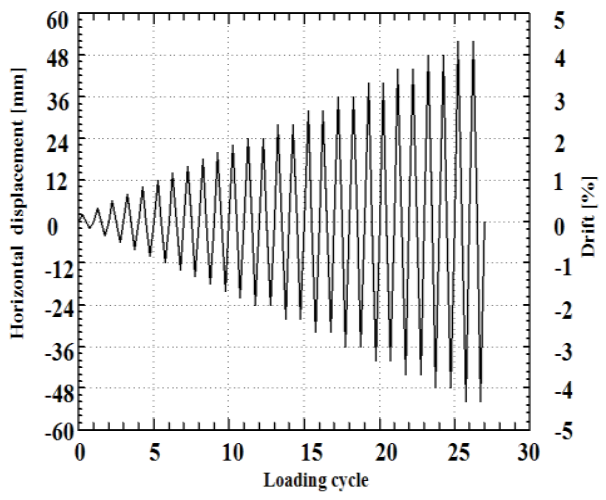

Figure 4 Loading system

Then the horizontal displacement of the specimen column reaches the limit and the test stops, as shown in Fig. 5 (d).

Common concrete columns do not appear cracks at the initial stage of loading, but with the increase of displacement, the number of cracks increases; when the ultimate bearing capacity is reached, the concrete on both sides is crushed. The failure mode is shown in Fig. 6 .

By comparing the failure phenomena, it can be seen that the failure of reinforced PP ECC columns has obvious precursors, while the deformation of ordinary reinforced concrete columns is obviously smaller than that of reinforced PP ECC columns. 


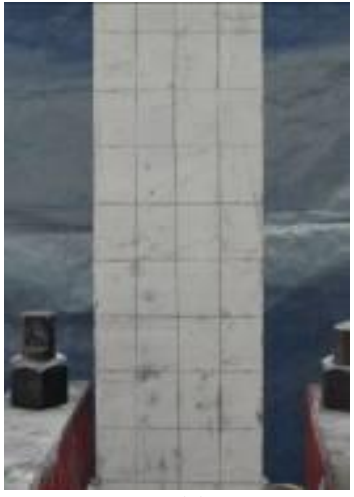

(a)

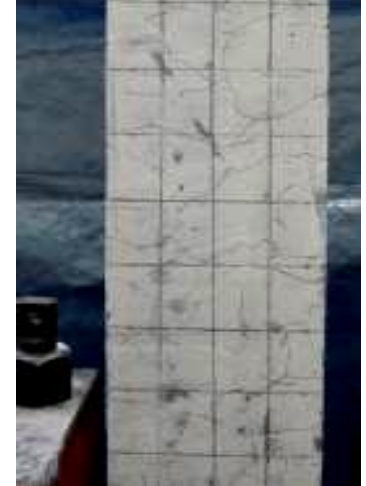

(b)

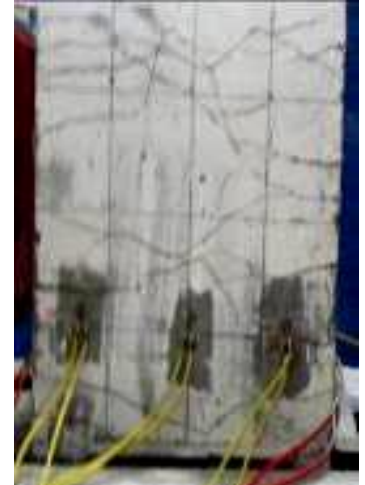

(c)

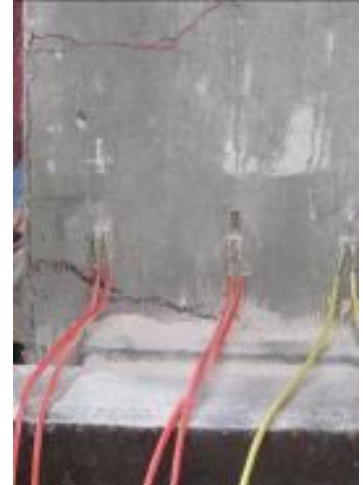

(d)

Figure 5 Damage drawing of PP ECC specimen

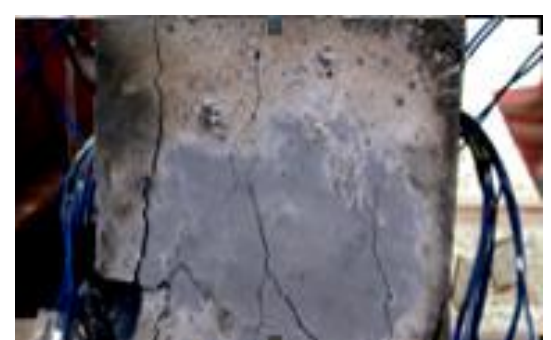

Figure 6 Failure modes of concrete specimen

\section{Analysis of Seismic Performance}

\section{1 $\mathrm{F}-\Delta$ hysteresis curve}

Fig. 7 shows the hysteretic curves of the specimens in the

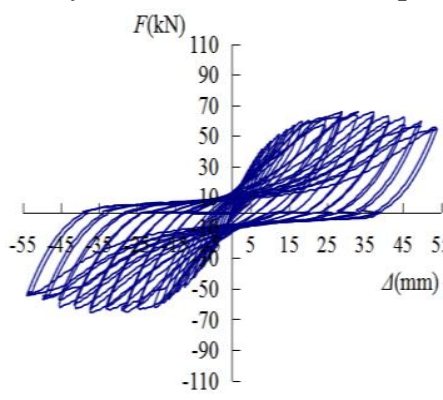

(a) $\mathrm{Cl}$

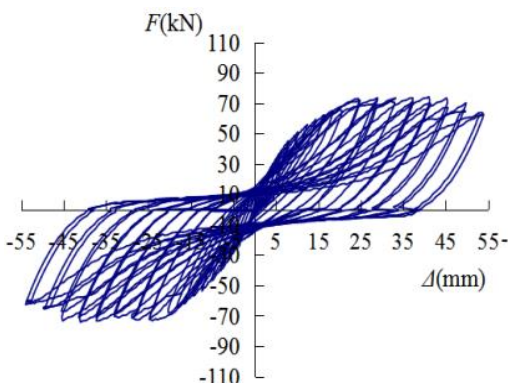

(d) $\mathrm{C} 4$

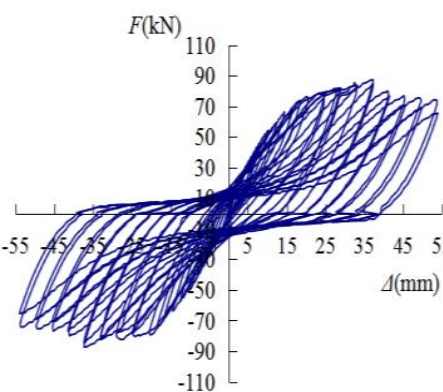

(b) $\mathrm{C} 2$ test. From Fig. 7 (a) $\sim$ (e), it can be seen that the hysteretic curve of reinforced PP ECC column is elastic in the early stage, and the hysteretic curve rises along a straight line; when the super-tough concrete column reaches yield, the curve shows a non-linear relationship; when the peak load is reached, the curve shows obvious ductility, and begins to develop from shuttle type to anti-S type, and the bearing capacity declines slowly.

From Fig. 7 (f), it can be seen that the hysteretic curve of ordinary concrete columns has no obvious pinching phenomenon, and the early stiffness is very large, showing a linear relationship. When the ultimate bearing capacity exceeds, the bearing capacity decreases rapidly.

Figure $7 F-\Delta$ hysteresis curve of PP ECC and concrete specimen 


\subsection{Hysteresis curve}

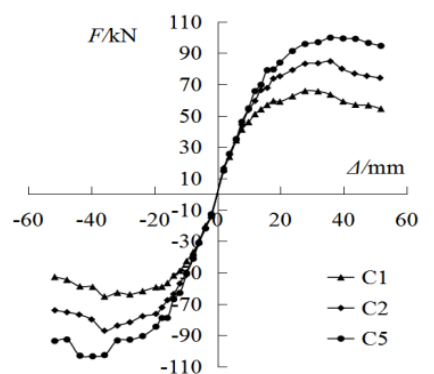

(a) Different reinforcement ratio

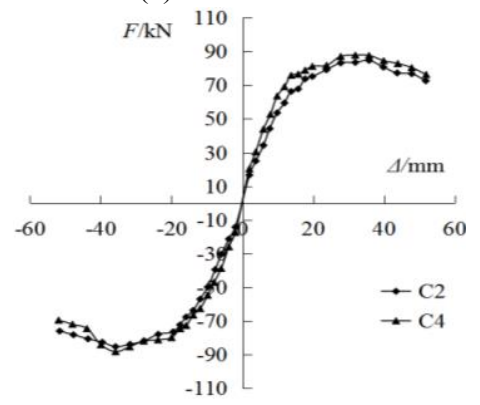

(c) Different PP content

Figure 8 Influences of different parameters on hysteresis curve

As shown in Figure 8, on the premise of fixed axial compression ratio, through the comparison of skeleton curves of reinforced PP ECC columns under different parameters, the following conclusions can be summarized:

- From Fig. 8 (a), it can be seen that the higher the reinforcement ratio, the higher the ultimate bearing capacity of PP ECC concrete columns, but the increasing range gradually decreases.

- From Fig. 8 (b), it can be seen that with the increase of age, the bearing capacity of PP ECC columns increases gradually, but the deformation capacity decreases.

- From Fig. 8 (c), it can be seen that the change of PP content between $1.5 \%$ and $2 \%$ has no obvious effect on the skeleton curve.

- From Fig. 8 (d), it can be seen that the deformation capacity of PP ECC columns is obviously better than that of ordinary concrete.

\subsection{Seismic energy dissipation}

In the hysteretic curve, the area enclosed in the ring after

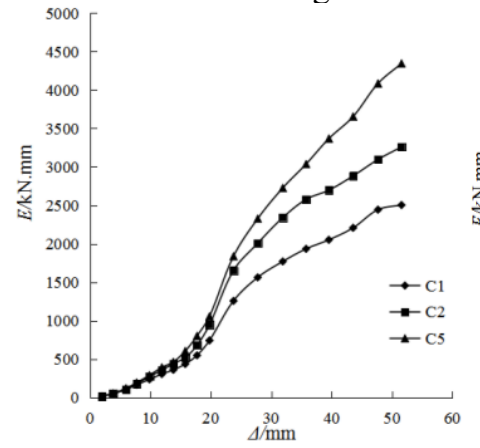

(a) Different reinforcement ratio
The skeleton curve ${ }^{[7]}$ is obtained by connecting the peak points of hysteretic curve.

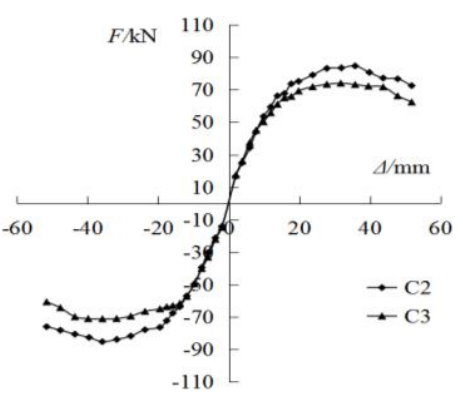

(b) Different age

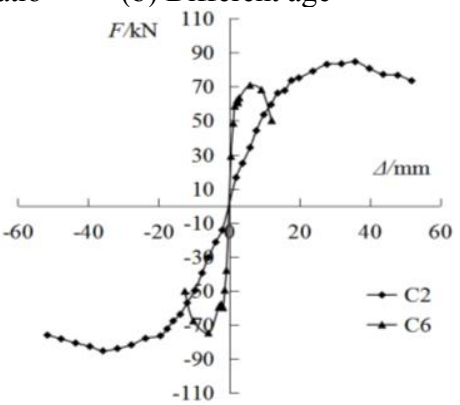

(d) Different concrete

one week of load cycle represents the seismic energy dissipation capacity of the specimen ${ }^{[8]}$. The total seismic energy dissipation $\mathrm{E}$ of each specimen is calculated by programming, and the comparison curve is drawn, as shown in Figure 9.

- From Fig. 9 (a), it can be seen that the seismic energy dissipation E of PP ECC columns increases gradually with the increase of reinforcement ratio.

- From Fig. 9 (b), it can be seen that the area of hysteretic curve of PP ECC columns of different ages is similar in the early stage of loading, but the energy dissipation performance of columns with higher age after yielding is higher than that of columns with lower age.

- From Fig. 9 (c), it can be seen that the change of PP content between $1.5 \%$ and $2 \%$ has no obvious effect on the seismic performance of the column.

- From Fig. 9 (d), it can be seen that the energy consumption of ordinary concrete columns is higher in the early stage, but lower in the later stage than that of PP ECC.

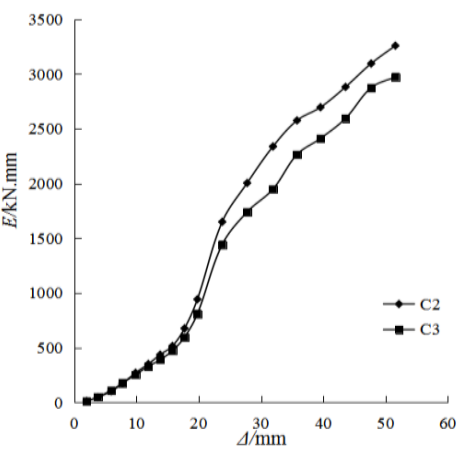

(b) Different age 


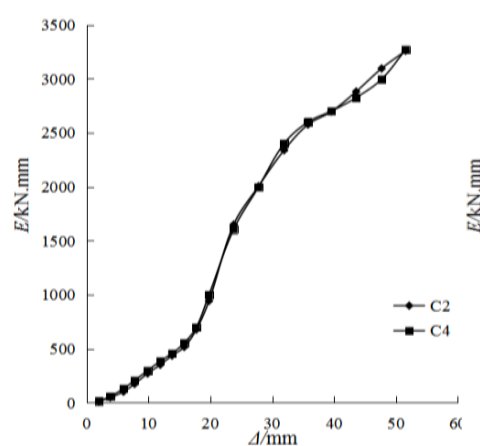

(c) Different PP content

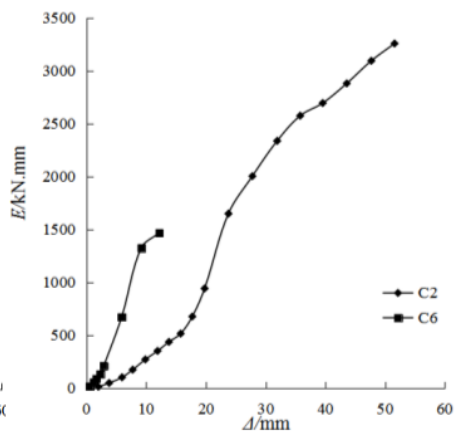

(d) Different concrete

Figure 9 Influences of parameters on dissipated energy

\section{Conclusion}

Through hysteretic test, it can be seen that reinforced PP ECC columns are obviously superior to ordinary concrete columns in bearing capacity, deformation capacity and energy dissipation capacity.

Within the range of test parameters, with the increase of reinforcement ratio, the bearing capacity of PP ECC columns increases gradually, and the seismic energy dissipation capacity increases gradually; the volume content of PP fiber has little effect on the seismic performance of columns in the range of $1.5 \% \sim 2.0 \%$; with the increase of age, the bearing capacity increases gradually, the deformation capacity decreases, while the seismic energy dissipation of columns in the age has a certain influence, but the influence is limited.

\section{References}

1. Victor C. Li. Introduction to Engineered Cementitious Composites (ECC)[M]. Springer Berlin Heidelberg:2019-05-01.

2. Yu.J.H, Dai Y and Song B.Design, Production and Mechanical Property of White Engineered Cementitious Composites. Advanced Materials Research,2010(97-101):1673-1676.

3. Yang. En.Hua,Li.V.C, Strain-hardening fiber cement optimization and component tailoring by means of a micromechanical model, Construction and Building Materials,2010.24(2):130-139.

4. Victor C. Li. Durability of Engineered Cementitious Composites (ECC) and Reinforced ECC (R/ECC) Structural Members[M]. Springer Berlin Heidelberg:2019-05-01.

5. Yi Bao, Mingfeng Xu,Daniel Soltan, Tian Xia, Albert Shih, Herek L. Clack, Victor C. Li. Three-Dimensional Printing Multifunctional Engineered Cementitious Composites (ECC) for Structural Elements[M]. Springer International Publishing:2018-08-30.

6. Ling-Zhi Li, Yang Bai,Ke-Quan Yu, Jiang-Tao Yu, Zhou-Dao Lu. Reinforced high-strength engineered cementitious composite (ECC) columns under eccentric compression: Experiment and theoretical model[J]. Engineering Structures,2019,198.
7. Jun Tian, Xiaowei Wu, Yu Zheng, Shaowei Hu, Yinfei Du, Wenwei Wang, Can Sun, Lifei Zhang. Investigation of interface shear properties and mechanical model between ECC and concrete[J]. Construction and Building Materials,2019,223.

8. Van der Geer, J., Hanraads, J.A.J., Lupton, R.A. (2010) The art of writing a scientific article. J. Sci. Commun., 163: 51-59. 